\title{
Molecular Imaging of Biomarkers in Breast Cancer
}

\author{
Gary A. Ulaner ${ }^{1,2}$, Chris C. Riedl ${ }^{1}$, Maura N. Dickler ${ }^{3}$, Komal Jhaveri $^{3}$, Neeta Pandit-Taskar ${ }^{1,2}$, and Wolfgang Weber ${ }^{1,2}$ \\ ${ }^{I}$ Department of Radiology, Memorial Sloan Kettering Cancer Center, New York, New York; ${ }^{2}$ Department of Radiology, \\ Weill Cornell Medical College, New York, New York; and ${ }^{3}$ Department of Medicine, Memorial Sloan Kettering Cancer Center, \\ New York, New York
}

\begin{abstract}
The success of breast cancer therapy is ultimately defined by clinical endpoints such as survival. It is valuable to have biomarkers that can predict the most efficacious therapies or measure response to therapy early in the course of treatment. Molecular imaging has a promising role in complementing and overcoming some of the limitations of traditional biomarkers by providing the ability to perform noninvasive, repeatable whole-body assessments. The potential advantages of imaging biomarkers are obvious and initial clinical studies have been promising, but proof of clinical utility still requires prospective multicenter clinical trials.
\end{abstract}

Key Words: molecular imaging; oncology; breast; PET/CT; biomarkers; breast cancer; ${ }^{18} \mathrm{~F}-\mathrm{FDG}$

J Nucl Med 2016; 57:53S-59S

DOI: 10.2967/jnumed.115.157909

$\mathbf{T}$ he success of breast cancer therapy is ultimately defined by clinical endpoints such as survival. However, measurement of these clinical endpoints requires prolonged follow-up, and they cannot guide the treatment of individual patients early in the course of therapy. Therefore, it is valuable to have biomarkers that can predict the most efficacious therapies or measure response to therapy early in the course of treatment. Biomarkers for predicting which therapies will be efficacious for breast cancer have traditionally been evaluated in tissue samples obtained from biopsy or surgery, whereas those assessing therapy response in the neoadjuvant and metastatic settings have traditionally been based on tumor size. Although proven to be valuable, these traditional biomarkers have several limitations. Molecular imaging has a promising role in complementing and overcoming some of the limitations of these established biomarkers. In this article, we review the role of tissue-based and molecular imaging biomarkers, including their advantages and limitations. We also discuss how molecular imaging biomarkers may guide individualized care of patients with breast cancer, as well as clinical trials of novel therapeutics.

\section{WHAT ARE BIOMARKERS?}

Biomarkers are often protein markers, such as prostate-specific antigen for the detection of prostate cancer (1), and genomic markers,

Received Jul. 23, 2015; revision accepted Aug. 26, 2015.

For correspondence or reprints contact: Gary Ulaner, Department of Radiology, Memorial Sloan Kettering Cancer Center, 1275 York Ave., New York, NY 10065. E-mail: ulanerg@mskcc.org

COPYRIGHT (C) 2016 by the Society of Nuclear Medicine and Molecular Imaging, Inc. such as epidermal growth factor receptor (EGFR) kinase mutations in non-small cell lung cancer, which predict response to EGFR kinase inhibitors (2). Examples such as these have led many scientists to limit their definition of biomarkers to tissue factors. For example, the National Cancer Institute has defined a biomarker as "a biologic molecule found in blood, other body fluids, or tissues that is a sign of a normal or abnormal process" (3). However, this definition would preclude important imaging biomarkers that are currently in use. Thus, it may be time to more broadly recognize biomarkers as measurable indicators of biologic processes, whether obtained from tissue, imaging, or other sources.

The treatment of breast cancer has been guided for many years by tissue-based biomarkers, including estrogen receptor (ER), progesterone receptor (PgR), and human epidermal growth factor receptor 2 (HER2) status (4). However, these biomarkers cannot capture the spatial heterogeneity of breast cancer that has been described at the intratumoral, intrametastatic, and intermetastatic levels (5-9). Furthermore, tumor cells undergo selection during therapy, which may change the dominant genotype and lead to treatment resistance (10). Studying this temporal heterogeneity of metastatic breast cancer with tissue-based biomarkers is a challenge because it requires sequential biopsies. Even if repeated biopsies are performed, they may not be informative because genetic changes may not occur at all metastatic sites at the same time. Molecular imaging studies can therefore complement tissuebased biomarkers, because they allow noninvasive assessment of disease throughout the entire body at a single or multiple times.

In discussing the clinical role of imaging-based biomarkers, it is also important to differentiate among prognostic, predictive, and pharmacodynamic biomarkers, because these require different approaches for validation (Table 1) (11). Prognostic markers assess intrinsically favorable or unfavorable biology of the disease but do not provide information to guide treatment $(11,12)$. For example, tumor stage can be considered a prognostic biomarker because the presence of nodal or distant metastases has been shown to strongly affect prognosis in patients with breast cancer. However, tumor stage itself does not help identify treatment that will be efficacious. Other examples of prognostic biomarkers in breast cancer include gene expression signatures that are associated with the risk for recurrence after primary therapy for breast cancer (e.g., Oncotype DX test [Genomic Health], PAM50 Breast Cancer Intrinsic Classifier [ARUP Laboratories], MammaPrint [Agendia], breast cancer profiling, or the ratio of HOXB13 to IL17BR [H/I ratio]). However, prognostic biomarkers do not predict whether a specific therapy will be successful.

In contrast, predictive markers correlate with the success of specific therapies and thus help select the optimal therapies for patient care. For example, ER and PgR status predict response to endocrine therapy (13), and HER2 amplification predicts response to HER2-targeted therapies such as trastuzumab (14). 
TABLE 1

Categories of Biomarkers

\begin{tabular}{lcc}
\hline \multicolumn{1}{c}{ Category } & Potential uses & Examples in breast cancer \\
\hline Prognostic & $\begin{array}{c}\text { Distinguish tumors with } \\
\text { intrinsically good or poor prognosis }\end{array}$ & $\begin{array}{c}\text { ER (4), HER2 (4), } \\
\text { Oncotype DX 21-gene panel (23), } \\
\text { circulating tumor cells (29) }\end{array}$ \\
\hline Predictive & $\begin{array}{c}\text { Determine which therapies will } \\
\text { be effective for individual patient } \\
\text { Pharmacodynamic }\end{array}$ & $\begin{array}{c}\text { ER (13), HER2 (14) } \\
\text { Determine dosing of novel } \\
\text { systemic therapies for clinical trials }\end{array}$ \\
\hline
\end{tabular}

Pharmacodynamic biomarkers measure the immediate effect of a drug on its target. This effect may not necessarily be associated with a favorable treatment outcome because the cancer cells may not be dependent on the targeted pathway or may develop resistance early in the course of therapy, but it provides proof-of-mechanism data during drug development. Pharmacodynamic markers that have been used in clinical trials include measurement of the inhibition of a signaling pathway or metabolic process in easily accessible tissues, such as peripheral blood mononuclear cells, skin, or plucked hair follicles (15).

Although it is important to understand these different classes of biomarkers, several overlaps exist. For example, ER expression is a favorable prognostic factor (4) as well as a predictive factor for success of endocrine therapies (13). HER2 expression is a negative prognostic factor in women who do not receive HER2-directed therapies but a positive predictive factor in women who do (14).

The goal of this article is to review currently used biomarkers in the management of breast cancer patients, discuss limitations of current biomarkers, and describe the potential for imaging-based biomarkers to provide additional and complementary information of clinical value.

\section{TISSUE- AND BLOOD-BASED PROGNOSTIC AND PREDICTIVE BIOMARKERS IN BREAST CANCER}

The American Society of Clinical Oncology (ASCO) evidencebased guidelines recommend that all patients with newly diagnosed breast cancer undergo evaluation of ER, PgR, and HER2 expression in tissue samples obtained from biopsy or surgery (4). Classification of breast tumors based on their ER, PgR, and HER2 expression status affects prognosis, predicts response to available systemic therapies, and helps tailor therapy for individual patients.

ER is a steroid hormone receptor found on approximately $70 \%$ of primary breast cancers. Breast cancer is considered to be ER-positive if as little as $1 \%$ of tumor nuclei express ER on immunohistochemistry (16). ER-positive breast cancers use estradiol as a main growth stimulus; thus, ER status is a critical index of sensitivity to endocrine therapies. Endocrine therapy with 5 y of adjuvant tamoxifen decreases the annual breast cancer death rate by more than $30 \%$ in ER-positive disease, whereas ER-negative disease demonstrates no benefit from this treatment except in the uncommon group of ER-negative but PgR-positive tumors (13). ER status also predicts response to neoadjuvant chemotherapy. ER-positive tumors have a lower likelihood of achieving a pathologic complete response (pCR) than ER-negative tumors (17).

The expression of $\mathrm{PgR}$ is strongly correlated to ER expression. Less than $1 \%$ of breast tumors are PgR-positive but ER-negative
(18). Patients with high levels of PgR expression in their breast cancer have better outcomes, but there is less predictive value of PgR level for hormonal treatment (19).

The oncogene HER2 encodes a human epidermal growth factor receptor. Amplification or mutation of this oncogene is found in approximately $20 \%$ of primary breast cancers (14). A breast cancer is considered to be HER2-positive if there is evidence of protein overexpression on immunohistochemistry or gene amplification on florescence in situ hybridization (20). HER2 was initially a prognostic biomarker, with HER2-positive malignancy prognostic of a worse outcome than HER2-negative malignancy (14). The development of trastuzumab, a monoclonal antibody targeted to HER2, made HER2 a powerful predictive biomarker as well. HER2 expression predicts successful trastuzumab therapy in early-stage (21) and metastatic (14) breast cancer. In current practice, patients with HER2-positive breast cancer receive specific targeted HER2 therapies that decrease the annual breast cancer death rate by one third $(14,21)$, whereas most patients with HER2-negative breast cancer do not benefit. The tremendous success of trastuzumab in patients with HER2-positive breast cancer has led to the development of additional HER2-targeted agents, such as pertuzumab, lapatinib, and adotrastuzumab emtansine (T-DM1) (22).

Multigene RNA profiling assays have been developed that may improve the prediction of outcomes over standard clinical and pathologic markers. For example, a reverse transcriptase-polymerase chain reaction assay of a 21-gene panel, the Oncotype Dx, was developed to predict the risk of distant metastases in patients with ER-positive, HER2-negative early-stage breast cancer who are receiving hormonal therapy with tamoxifen (23). A high recurrence score on this panel predicts a benefit from the addition of chemotherapy in these patients (24). ASCO guidelines include the Oncotype Dx recurrence score in their recommendations for nodenegative, ER-positive, HER2-negative patients. An enzyme-linked immunosorbent assay for urokinase plasminogen activator and plasminogen activator inhibitor-1 (PAI-1) may also help determine the risk of disease recurrence in patients with node-negative breast cancer and thus inform the necessity of chemotherapy (25). However, large tissue sections are required, and the value of the assay using tissue from limited core-needle biopsies has not been confirmed (26).

The rapid development of sequencing technologies has resulted in newer blood analyses of solid tumors based on circulating tumor cells (CTCs) and circulating tumor DNA. CTCs are rare cancer cells found in the peripheral blood $(27,28)$ that are believed to play a role in tumor progression. There is a high level of evidence of the value of CTCs as a prognostic biomarker for breast cancer (29). In a retrospective study of 115 patients with metastatic 
breast cancer, monitoring CTCs in the blood was superior for monitoring tumor response to therapy than radiologic response assessment using ${ }^{18} \mathrm{~F}$-FDG PET/CT (30). A prospective multicenter study (SWOG S0500) (31) has confirmed the outstanding ability of CTC measurements for predicting survival of patients with metastatic breast cancer. In 595 patients, median survival of patients without and with persistent tumor cells $3 \mathrm{wk}$ after the start of chemotherapy was 35 and 13 mo, respectively (31). Circulating tumor DNA are fragments of cell-free DNA in the blood that contain tumor-specific sequence alterations (32). Recent investigations demonstrate that circulating tumor DNA has the potential to be a highly sensitive biomarker for breast cancer (32). An inherent advantage of CTCs and circulating DNA is the ability to test the samples for acquired resistance mechanisms, such as specific mutations. This may allow in the future not only the identification of nonresponders but also the rational selection of second-line therapies. Standardization of assays for CTCs is currently ongoing. If successful, complex, observer-dependent radiology studies may be replaced by a simple blood test with an automated, objective analysis that requires only a few milliliters of venous blood. In addition to CTCs and tumor DNA, disseminated tumor cells in the bone marrow have also been shown in a recently published study to have value for predicting the outcome of patients undergoing chemotherapy (33). That study investigated the prognostic value of disseminated tumor cells in patients with early breast cancer: 1,066 patients with disseminated tumor cells in the bone marrow after adjuvant therapy received 6 additional cycles of docetaxel chemotherapy. Patients whose bone marrow remained positive for disseminated tumor cells after docetaxel therapy had a high relapse rate of $46 \%$, whereas the relapse rate was only $8.8 \%$ for patients with negative bone marrow.

\section{POTENTIAL OF PROGNOSTIC AND PREDICTIVE MOLECULAR IMAGING BIOMARKERS}

Although these established biomarkers have proven to be valuable and are in wide clinical use, they still have limitations. Importantly, assays for receptor expression and gene profiling are typically performed on individual tissue samples obtained at the time of biopsy or surgery. Thus, in addition to the invasive nature of the tissue collection, only one or a small number of sites of disease are typically sampled. However, growing evidence suggests that biomarker expression may change temporally and spatially, resulting in metastases with biomarker expression different from the primary tumor, as well as varying biomarker expression among different metastases $(7,8)$. Inaccurate knowledge of receptor status due to tumor heterogeneity may lead to suboptimal treatment of metastatic breast cancer. Molecular imaging, such as with PET/CT, would allow noninvasive evaluation of all lesions - both primary and secondary —in a patient during a single examination and would potentially visualize tumor heterogeneity.

Perhaps the prototype for molecular imaging-based predictive biomarkers in breast cancer is ER imaging with $16 \alpha-{ }^{18} \mathrm{~F}$-fluoro$17 \beta$-estradiol $\left({ }^{18} \mathrm{~F}\right.$-FES). Because $20 \%$ of patients may demonstrate heterogeneity of ER expression at different sites of malignancy $(5,6)$, molecular imaging of ER status has been actively pursued. Among several estrogens labeled with various radionuclides, ${ }^{18} \mathrm{~F}-\mathrm{FES}$ is by far the most extensively studied (34). ${ }^{18} \mathrm{~F}-\mathrm{FES}$ uptake correlates strongly with ER expression as measured by immunohistochemistry (35) and successfully evaluates ER heterogeneity in vivo (36). Similar to tissue-based immunohistochemistry for ER, ${ }^{18} \mathrm{~F}-\mathrm{FES}$ uptake predicts response to endocrine therapy, with poor ${ }^{18} \mathrm{~F}-\mathrm{FES}$ uptake predicting a lack of response to endocrine therapy (37-39). Thus, molecular imaging of ER may provide additive predictive value over tissue assays for ER.

Given the success of HER2-targeted therapy, molecular imaging of the HER2 biomarker has also been an active area of development. Multiple radiolabeled HER2 antibodies and antibody fragments have been produced for both SPECT and PET (40-43). These tracers have been used to demonstrate HER2-specific uptake in patients with HER2-positive metastatic breast cancer. Because PET imaging allows higher resolution and signal-to-noise ratios than SPECT, and antibodies require multiple days for blood pool clearance and tumor uptake, relatively longer half-life PET isotopes such as ${ }^{89} \mathrm{Zr}$ (half-life, $78 \mathrm{~h}$ ) have demonstrated particularly high-quality imaging (44). Preliminary results using ${ }^{89} \mathrm{Zr}$-trastuzumab PET/CT in patients with HER2positive metastatic breast cancer suggest that ${ }^{89} \mathrm{Zr}$-trastuzumab uptake helps predict response to HER2-targeted therapy (45).

\section{ASSESSMENT OF DISEASE RESPONSE TO NEOADJUVANT THERAPY}

Neoadjuvant (i.e., preoperative) chemotherapy is used in locally advanced, nonmetastatic breast cancer to decrease the size of the primary tumor and local lymph nodes, potentially allowing a decreased extent of surgical intervention (46). The effectiveness of neoadjuvant chemotherapy can be assessed by pathologic analysis of the posttherapy tumor specimen. A pCR often correlates with improved outcomes (47). However, note that the definition of pCR among studies is not uniform, with pCR variably defined as the absence of both invasive and in situ cancer in both breast and nodes, the absence of invasive cancer but allowing the presence of in situ cancer in breast and nodes, or the absence of invasive cancer in the breast irrespective of in situ or nodal disease. This lack of uniformity makes comparison of studies more difficult. In addition, rates of pCR and implications of pCR depend on the receptor status of the primary tumor $(48,49)$. And despite some support for using pCR to predict improved outcomes, a large metaanalysis evaluating $\mathrm{pCR}$, comprising nearly 12,000 patients, did not find pCR to be a surrogate endpoint for improvement of overall survival by neoadjuvant chemotherapy (50).

Because even pathologic evaluation of neoadjuvant therapy response is limited, opportunities exist for the development of molecular imaging biomarkers to predict responses early after treatment initiation. An in-depth analysis of ${ }^{18} \mathrm{~F}$-FDG PET/CT for the prediction of neoadjuvant response evaluation in patients with breast cancer is detailed in a separate article (51) in this issue; however, a few concepts are worth emphasizing here. First, the ability of ${ }^{18}$ F-FDG PET/CT to predict pCR may depend on the ER and HER2 receptor status of the primary breast malignancy (49). Second, the metric for quantifying ${ }^{18}$ F-FDG uptake, such as $\mathrm{SUV}_{\max }$ or metabolic tumor volume, that optimally determines neoadjuvant response may differ for tumors of different receptor status (50). Finally, because low levels of residual tumor may not demonstrate ${ }^{18}$ F-FDG avidity that is apparent on PET, it may be challenging to develop an application of ${ }^{18} \mathrm{~F}$-FDG PET that alters the current standard of pathologic assessment after completion of neoadjuvant therapy to provide clinical utility.

\section{BIOMARKERS FOR MONITORING DISEASE RESPONSE IN METASTATIC BREAST CANCER}

Clinical methods for monitoring disease response are measurements of changes in tumor size and serum markers. Although used extensively in clinical practice, neither serum markers nor changes 
in tumor size have supporting evidence that is as strong for predicting survival as the use of the predictive markers ER and HER2.

The 2007 ASCO recommendations include the use of the serum markers cancer antigen 15-3, cancer antigen 27-29, and carcinoembryonic antigen for limited indications (4). Using tumor markers for evaluating tumor response offers advantages, including minimal invasiveness from blood samples, low cost, and ready availability. However, not all breast malignancies are detectable by tumor markers, and markers are often not specific for malignancy (4), with benign conditions such as ovarian cysts, thyroid disorders, hepatitis, and sarcoidosis resulting in false-positives (53). Therefore, ASCO guidelines recommend that serum markers not be used alone to monitor disease status, but in combination with imaging, history, and physical examination.

Change in tumor size based on anatomic imaging is the most commonly used biomarker for monitoring response of most solid tumors, including breast cancer. Indeed, the Food and Drug Administration may use progression-free survival, determined from measurements of tumor sizes, as a surrogate endpoint for overall survival when considering approval of cancer therapies (54). Multiple guidelines have been developed for standardized measurement of solid tumor masses as a means of monitoring disease response, including the World Health Organization guidelines in 1981 (55), RECIST in 2000 (56), and RECIST 1.1 in 2009 (57). All these guidelines use uni- or bidimensional linear measurements of tumor masses that are, of course, volumetric objects. Although this approach is widely used, it is important to consider its many limitations. First, after systemic or radiation therapy, it is often difficult to distinguish anatomically active tumor from posttherapy changes or scarring, which is the basis on which metabolic imaging such as ${ }^{18}$ F-FDG PET/CT takes precedence over anatomic imaging in the evaluation of posttreatment lymphoma (58). Second, linear measurements of 3-dimensional objects often have substantial intra- and interobserver variability. For example, variability in measurement of non-small cell lung cancers resulted in a 30\% rate of inconsistencies in interobserver RECIST classifications (59). Third, classic cytotoxic chemotherapies cause cell death, which may be measurable as decreases in tumor size; however, newer immunologic systemic therapies may effectively treat patients and result in improved outcomes despite tumors initially being stable or even increasing in size. Examples include imatinib in gastrointestinal stromal tumors (60), erlotinib in non-small cell lung cancer (61), and ipilimumab in melanoma (62). Thus, whereas it may seem logical that therapies that decrease tumor size would lead to improved outcomes, and data support this statement in hematologic malignancies such as lymphoma (63), data for solid tumors are not particularly strong (64). In a comprehensive metaanalysis of patients with breast cancer, including 11 randomized trials and nearly 4,000 patients, the correlation between improvement in tumor response rates, as measured by World Health Organization size criteria, and overall survival improvement from chemotherapy was 0.57 (95\% confidence interval, -0.31 to 1.44$)$, which "indicated a loose and imprecise estimated association" between these variables (65). The authors of the metaanalysis concluded that tumor response measured by size criteria was not shown to be a good surrogate biomarker for overall survival improvement in patients with breast cancer (65).

\section{POTENTIAL OF MOLECULAR IMAGING FOR MONITORING DISEASE RESPONSE IN METASTATIC BREAST CANCER}

Because of the limitations of monitoring disease response in solid tumors with serum markers or size criteria, there are ample opportunities for molecular imaging. Although multiple molecular approaches are currently in development, including novel MR sequences, contrast-enhanced ultrasound, optical imaging, and nanoparticles, ${ }^{18} \mathrm{~F}$-FDG PET is by far the most clinically investigated and used $(66,67) .{ }^{18}$ F-FDG PET has demonstrated several advantages in areas in which anatomic imaging has limitations. ${ }^{18} \mathrm{~F}$-FDG has a better ability to differentiate active tumor and posttherapy scarring (58). Measurements of ${ }^{18} \mathrm{~F}-\mathrm{FDG}$ avidity, such as $\mathrm{SUV}_{\max }$, have been found to be more reproducible than measurements of tumor size (68). Finally, ${ }^{18}$ F-FDG avidity can better discriminate chemotherapy effects of newer targeted therapies than size measurements can $(60,62)$.

The combination of ${ }^{18} \mathrm{~F}-\mathrm{FDG}$ PET with $\mathrm{CT}$ in modern hybrid PET/CT scanners has allowed better differentiation of pathologic ${ }^{18}$ F-FDG avidity from false-positive ${ }^{18}$ F-FDG avidity (69) and has led to increasing investigation of ${ }^{18} \mathrm{~F}$-FDG PET/CT for monitoring treatment response. Although a comprehensive analysis of response evaluation by ${ }^{18} \mathrm{~F}$-FDG PET/CT in patients with metastatic breast cancer is discussed in a separate article in this issue (70), a few concepts are worth noting here.

First, ${ }^{18} \mathrm{~F}-\mathrm{FDG}$ PET/CT has particular advantages for the evaluation of osseous metastases (Fig. 1), where changes in ${ }^{18} \mathrm{~F}-\mathrm{FDG}$ avidity more accurately evaluate response to treatment than anatomic or morphologic findings on CT $(71,72)$. The evaluation of osseous metastases on anatomic imaging is so severely restricted because of the inability to distinguish treatment-related sclerotic changes from progression that osseous lesions are not eligible to be selected as target lesions on RECIST $(56,57)$ unless a measurable soft-tissue component is present.

Second, breast cancer is a heterogeneous disease; thus, ${ }^{18} \mathrm{~F}-\mathrm{FDG}$ PET/CT may not have the same value in all breast cancers. The ER and HER2 receptor status of breast malignancies influences changes in the ${ }^{18}$ F-FDG avidity of these cancers after treatment $(49,52)$ and should therefore be considered when evaluating treatment response with ${ }^{18}$ F-FDG PET/CT.

Third, because both primary (73) and metastatic (74) lesions from invasive lobular carcinoma are less apparent on ${ }^{18} \mathrm{~F}-\mathrm{FDG}$ PET than

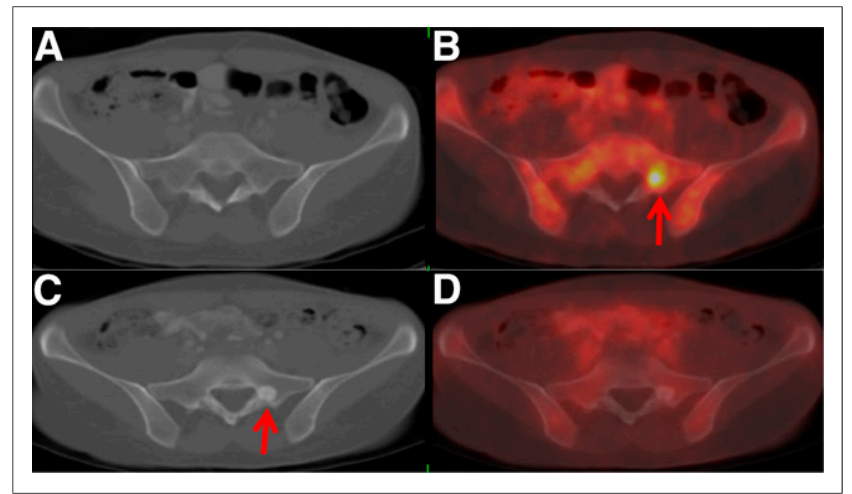

FIGURE 1. 41-y-old woman with primary ER-positive, HER2-negative invasive ductal breast carcinoma. Axial CT (A) and fused ${ }^{18} \mathrm{~F}-\mathrm{FDG}$ PET/ CT (B) images from first examination demonstrate ${ }^{18} \mathrm{~F}-\mathrm{FDG}$-avid osseous foci (arrow) without CT correlates. Biopsy demonstrated osseous metastases. After systemic therapy, axial CT (C) and fused ${ }^{18}$ F-FDG PET/ CT (D) images demonstrated resolution of ${ }^{18} \mathrm{~F}$-avid foci but revealed new sclerotic osseous lesions on CT (arrow). Without ${ }^{18}$ F-FDG PET, new sclerotic lesions could be mistaken for new osseous metastases, but inclusion of ${ }^{18} \mathrm{~F}$-FDG PET indicated that new sclerotic lesions are consistent with treated disease. 
comparable lesions in patients with the more common invasive ductal carcinoma, tumor histology may be another important factor to consider.

Fourth, metabolic responses in breast cancer are often heterogeneous (75). Within a single patient, there are often both increasing and decreasing lesions on a given follow-up examination (Fig. 2), which may be considered a mixed response. The concept of mixed response is not addressed in current anatomic response criteria such as RECIST $(56,57)$, and the implications of mixed metabolic responses have not been determined.

Finally, increasing evidence suggests that ${ }^{18} \mathrm{~F}-\mathrm{FDG}$ PET/CT is a more accurate method of evaluating treatment response in patients with metastatic breast cancer than anatomic response criteria $(76,77)$. Will this translate to improved prediction of patient outcomes? An abstract presented at the 2015 ASCO annual meeting of a retrospective trial comparing treatment response in 71 patients with metastatic breast cancer by ${ }^{18} \mathrm{~F}$-FDG PET/CT versus contrast-enhanced CT suggests that progression-free survival was better predicted by ${ }^{18}$ F-FDG PET/CT (78), but prospective data are sparse. A recent prospective study of the use of the phosphoinositide-3-kinase inhibitor buparlisib with letrozole in ER-positive, HER2-negative metastatic breast cancer demonstrated that lack of metabolic response by ${ }^{18} \mathrm{~F}-\mathrm{FDG} \mathrm{PET} / \mathrm{CT}$ at $2 \mathrm{wk}$ was associated with treatment failure and rapid disease progression (79). A study from the Translational Breast Cancer Research Consortium that includes prospective evaluation of ${ }^{18}$ F-FDG PET/CT response to lapatinib and trastuzumab in HER2-positive patients is in press (80). Further prospective studies are needed to establish ${ }^{18} \mathrm{~F}$-FDG PET/CT as a proven biomarker of response under REporting recommendations for tumor MARKers (REMARK) guidelines (81). The standardization of PET response

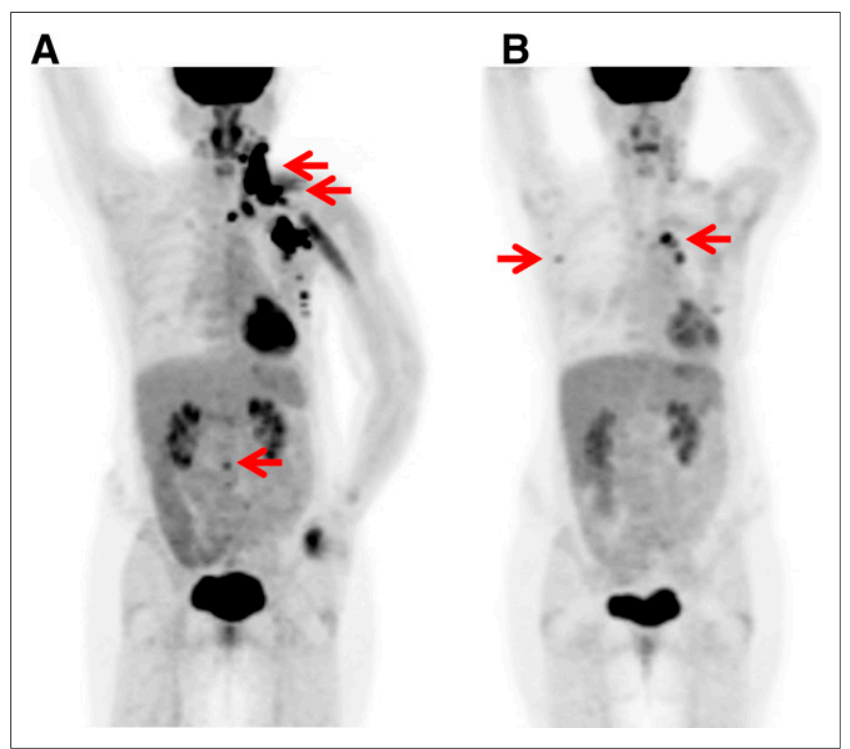

FIGURE 2. 52-y-old woman with metastatic ER-negative, HER2-positive breast cancer. (A) ${ }^{18} \mathrm{~F}-\mathrm{FDG}$ maximum-intensity-projection image demonstrates ${ }^{18} \mathrm{~F}-\mathrm{FDG}$-avid malignancy (arrows) in left neck, axilla, and chest wall, as well as focus in L2 vertebra. (B) After 8 wk of systemic therapy with paclitaxel, trastuzumab, and pertuzumab, these lesions resolved, but new ${ }^{18} \mathrm{~F}-\mathrm{FDG}-$ avid mediastinal and right axillary nodes appeared (arrows), which were biopsy-proven to be new metastases. Combination of both decreasing and new lesions after therapy is example of metabolic mixed response, which is common in metastatic breast cancer and suggests tumor inhomogeneity.

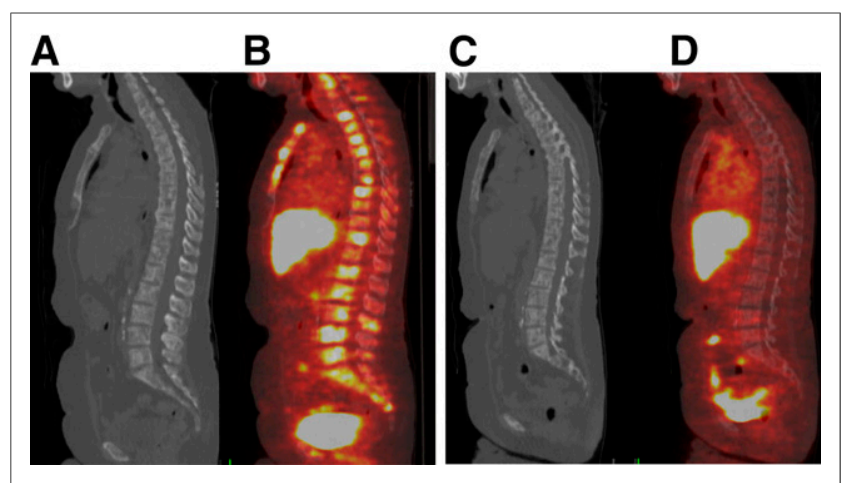

FIGURE 3. 67-y-old woman with ER-positive, HER2-negative metastatic breast cancer. Initial sagittal CT (A) and fused ${ }^{18} \mathrm{~F}-\mathrm{FES} \mathrm{PET/CT} \mathrm{(B)} \mathrm{images}$ demonstrate multifocal ${ }^{18} \mathrm{~F}$-FES-avid osseous metastases. ${ }^{18} \mathrm{~F}$-FES in liver and bowel is physiologic. (C and $\mathrm{D})$ After therapy with novel ER antagonist and degrader GDC-0810, ${ }^{18} \mathrm{~F}-\mathrm{FES}$ avidity in osseous metastases resolves, demonstrating ER engagement and abolished ER availability.

criteria in the PERCIST guidelines provides a framework for future evaluations $(81)$

\section{ROLE OF MOLECULAR IMAGING BIOMARKERS IN CLINICAL TRIALS OF NOVEL THERAPEUTICS}

Dosing of systemic therapies in clinical trials is usually determined by the maximum tolerated dose in phase I studies. But for targeted therapeutics, the optimal dose may be less dependent on what dose causes intolerable side effects and more appropriately determined by a biomarker that identifies successful target binding. ${ }^{18} \mathrm{~F}-\mathrm{FES}$ has played an integral role in the design of clinical trials for these agents; it has been used as a pharmacodynamic biomarker to monitor ER engagement by novel ER-targeted therapies and to determine the optimal dosage for these novel therapies.

In a proof-of-concept study, ${ }^{18} \mathrm{~F}-\mathrm{FES}$ PET/CT was used to demonstrate target engagement by fulvestrant, an ER antagonist, and to select the dose of fulvestrant needed to abolish ER availability (study registered in clinicaltrials.gov as NCT01377324) (83). Similar work has been successfully applied to the ER antagonist and degrader GDC-0810 (Fig. 3) (84).

\section{LIMITATIONS AND CHALLENGES}

The use of molecular imaging biomarkers is in its early stages, and prospective data are generally lacking. Before receiving acceptance as a clinically valuable biomarker, molecular imaging techniques face several challenges, including documentation of test reproducibility in multiple clinical trials, demonstration of a strong correlation between the test and a clinical outcome, and, perhaps most challenging, evidence that use of the test results in improved outcomes (85). Meeting these challenges will require multiinstitutional collaborations of defined biomarkers; carefully designed studies with prospectively defined study endpoints; and standardized data acquisition, data analysis, and reporting of results.

\section{CONCLUSION}

Few breast cancer biomarkers have met the high bar to prove clinical utility, such as ER, PgR, and HER2 assays for predicting effectiveness of systemic therapy and the Oncotype DX 21-gene 
panel for estimating risk of disease recurrence and predictive benefit of chemotherapy. Spatial and temporal tumoral heterogeneity limit these tissue-based assays. Molecular imaging techniques provide the ability to perform noninvasive, repeatable wholebody assessments and thus have the potential to play critical roles as prognostic, predictive, or pharmacodynamic biomarkers. The potential advantages of imaging biomarkers are obvious, and initial clinical studies have been promising. But proof of the clinical utility of imaging biomarkers still requires prospective multicenter clinical trials.

\section{DISCLOSURE}

This study was funded by Susan G. Komen for the Cure (Gary A. Ulaner). No other potential conflict of interest relevant to this article was reported.

\section{REFERENCES}

1. Balk SP, Ko YJ, Bubley GJ. Biology of prostate-specific antigen. J Clin Oncol. 2003;21:383-391.

2. Pao W, Miller VA, Politi KA, et al. Acquired resistance of lung adenocarcinomas to gefitinib or erlotinib is associated with a second mutation in the EGFR kinase domain. PLoS Med. 2005;2:e73.

3. Henry NL, Hayes DF. Cancer biomarkers. Mol Oncol. 2012;6:140-146.

4. Harris L, Fritsche H, Mennel R, et al. American Society of Clinical Oncology 2007 update of recommendations for the use of tumor markers in breast cancer. J Clin Oncol. 2007;25:5287-5312.

5. Hilton JF, Amir E, Hopkins S, et al. Acquisition of metastatic tissue from patients with bone metastases from breast cancer. Breast Cancer Res Treat. 2011;129:761-765.

6. Amir E, Miller N, Geddie W, et al. Prospective study evaluating the impact of tissue confirmation of metastatic disease in patients with breast cancer. $J$ Clin Oncol. 2012;30:587-592.

7. Niikura N, Liu J, Hayashi N, et al. Loss of human epidermal growth factor receptor 2 (HER2) expression in metastatic sites of HER2-overexpressing primary breast tumors. J Clin Oncol. 2012;30:593-599.

8. Hoefnagel LD, van de Vijver MJ, van Slooten HJ, et al. Receptor conversion in distant breast cancer metastases [abstract]. Breast Cancer Res. 2010;12:R75.

9. Chang HJ, Han SW, Oh DY, et al. Discordant human epidermal growth factor receptor 2 and hormone receptor status in primary and metastatic breast cancer and response to trastuzumab. Jpn J Clin Oncol. 2011;41:593-599.

10. Normanno N, Di Maio M, De Maio E, et al. Mechanisms of endocrine resistance and novel therapeutic strategies in breast cancer. Endocr Relat Cancer. 2005;12:721-747.

11. Sawyers CL. The cancer biomarker problem. Nature. 2008;452:548-552.

12. Mankoff DA, Pryma DA, Clark AS. Molecular imaging biomarkers for oncology clinical trials. J Nucl Med. 2014;55:525-528.

13. Early Breast Cancer Trialists' Collaborative Group (EBCTCG). Effects of chemotherapy and hormonal therapy for early breast cancer on recurrence and 15-year survival: an overview of the randomised trials. Lancet. 2005;365:1687-1717.

14. Slamon DJ, Leyland-Jones B, Shak S, et al. Use of chemotherapy plus a monoclonal antibody against HER2 for metastatic breast cancer that overexpresses HER2. N Engl J Med. 2001;344:783-792.

15. Ang JE, Kaye S, Banerji U. Tissue-based approaches to study pharmacodynamic endpoints in early phase oncology clinical trials. Curr Drug Targets. 2012;13:1525-1534.

16. Hammond ME, Hayes DF, Dowsett M, et al. American Society of Clinical Oncology/College of American Pathologists guideline recommendations for immunohistochemical testing of estrogen and progesterone receptors in breast cancer. J Clin Oncol. 2010;28:2784-2795.

17. Colleoni M, Viale G, Zahrieh D, et al. Chemotherapy is more effective in patients with breast cancer not expressing steroid hormone receptors: a study of preoperative treatment. Clin Cancer Res. 2004;10:6622-6628.

18. Viale G, Regan MM, Maiorano E, et al. Prognostic and predictive value of centrally reviewed expression of estrogen and progesterone receptors in a randomized trial comparing letrozole and tamoxifen adjuvant therapy for postmenopausal early breast cancer: BIG 1-98. J Clin Oncol. 2007;25:3846-3852.
19. Dowsett M, Houghton J, Iden C, et al. Benefit from adjuvant tamoxifen therapy in primary breast cancer patients according oestrogen receptor, progesterone receptor, EGF receptor and HER2 status. Ann Oncol. 2006;17:818-826.

20. Wolff AC, Hammond ME, Hicks DG, et al. Recommendations for human epidermal growth factor receptor 2 testing in breast cancer: American Society of Clinical Oncology/College of American Pathologists clinical practice guideline update. J Clin Oncol. 2013;31:3997-4013.

21. Romond EH, Perez EA, Bryant J, et al. Trastuzumab plus adjuvant chemotherapy for operable HER2-positive breast cancer. $N$ Engl J Med. 2005;353:1673-1684.

22. Singh JC, Jhaveri K, Esteva FJ. HER2-positive advanced breast cancer: optimizing patient outcomes and opportunities for drug development. $\mathrm{Br} \mathrm{J}$ Cancer. 2014;111:1888-1898.

23. Paik S, Shak S, Tang G, et al. A multigene assay to predict recurrence of tamoxifentreated, node-negative breast cancer. N Engl J Med. 2004;351:2817-2826.

24. Paik S, Tang G, Shak S, et al. Gene expression and benefit of chemotherapy in women with node-negative, estrogen receptor-positive breast cancer. J Clin Oncol. 2006;24:3726-3734.

25. Look MP, van Putten WL, Duffy MJ, et al. Pooled analysis of prognostic impact of urokinase-type plasminogen activator and its inhibitor PAI-1 in 8377 breast cancer patients. J Ntl Cancer Inst. 2002;94:116-128.

26. Schmitt M, Sturmheit AS, Welk A, Schnelldorfer C, Harbeck N. Procedures for the quantitative protein determination of urokinase and its inhibitor, PAI-1, in human breast cancer tissue extracts by ELISA. Methods Mol Med. 2006;120: $245-265$.

27. De Mattos-Arruda L, Cortes J, Santarpia L, et al. Circulating tumour cells and cellfree DNA as tools for managing breast cancer. Nat Rev Clin Oncol. 2013;10:377-389.

28. Ignatiadis M, Dawson SJ. Circulating tumor cells and circulating tumor DNA for precision medicine: dream or reality? Ann Oncol. 2014;25:2304-2313.

29. Cristofanilli M, Budd GT, Ellis MJ, et al. Circulating tumor cells, disease progression, and survival in metastatic breast cancer. N Engl J Med. 2004;351:781-791.

30. De Giorgi U, Valero V, Rohren E, et al. Circulating tumor cells and ${ }^{18} \mathrm{~F}$-fluorodeoxyglucose positron emission tomography/computed tomography for outcome prediction in metastatic breast cancer. J Clin Oncol. 2009;27:3303-3311.

31. Smerage JB, Barlow WE, Hortobagyi GN, et al. Circulating tumor cells and response to chemotherapy in metastatic breast cancer: SWOG S0500. J Clin Oncol. 2014;32:3483-3489.

32. Dawson SJ, Tsui DW, Murtaza M, et al. Analysis of circulating tumor DNA to monitor metastatic breast cancer. N Engl J Med. 2013;368:1199-1209.

33. Naume B, Synnestvedt M, Falk RS, et al. Clinical outcome with correlation to disseminated tumor cell (DTC) status after DTC-guided secondary adjuvant treatment with docetaxel in early breast cancer. J Clin Oncol. 2014;32:3848-3857.

34. van Kruchten M, Glaudemans AW, de Vries EF, et al. PET imaging of estrogen receptors as a diagnostic tool for breast cancer patients presenting with a clinical dilemma. J Nucl Med. 2012;53:182-190.

35. Peterson LM, Mankoff DA, Lawton T, et al. Quantitative imaging of estrogen receptor expression in breast cancer with PET and ${ }^{18} \mathrm{~F}$-fluoroestradiol. $\mathrm{J} \mathrm{Nucl}$ Med. 2008;49:367-374.

36. Kurland BF, Peterson LM, Lee JH, et al. Between-patient and within-patient (site-to-site) variability in estrogen receptor binding, measured in vivo by ${ }^{18}$ F-fluoroestradiol PET. J Nucl Med. 2011;52:1541-1549.

37. Mortimer JE, Dehdashti F, Siegel BA, Katzenellenbogen JA, Fracasso P, Welch MJ. Positron emission tomography with $2-{ }^{18} \mathrm{~F}$-fluoro-2-deoxy-D-glucose and $16 \alpha-{ }^{18} \mathrm{~F}$-fluoro$17 \beta$-estradiol in breast cancer: correlation with estrogen receptor status and response to systemic therapy. Clin Cancer Res. 1996;2:933-939.

38. Linden HM, Stekhova SA, Link JM, et al. Quantitative fluoroestradiol positron emission tomography imaging predicts response to endocrine treatment in breast cancer. J Clin Oncol. 2006;24:2793-2799.

39. Peterson LM, Kurland BF, Schubert EK, et al. A phase 2 study of $16 \alpha-{ }^{18}$ F-fluoro$17 \beta$-estradiol positron emission tomography (FES-PET) as a marker of hormone sensitivity in metastatic breast cancer (MBC). Mol Imaging Biol. 2014;16:431-440.

40. Dijkers EC, de Vries EG, Kosterink JG, Brouwers AH, Lub-de Hooge MN. Immunoscintigraphy as potential tool in the clinical evaluation of HER2/neu targeted therapy. Curr Pharm Des. 2008;14:3348-3362.

41. Mendler CT, Gehring T, Wester HJ, Schwaiger M, Skerra A. ${ }^{89} \mathrm{Zr}$ - versus ${ }^{124} \mathrm{I}$-labeled $\alpha$ HER2 fab with optimized plasma-half life for high-contrast tumor imaging in vivo. J Nucl Med. 2015;56:1112-1118.

42. Dijkers EC, Kosterink JG, Rademaker AP, et al. Development and characterization of clinical-grade ${ }^{89} \mathrm{Zr}$-trastuzumab for HER2/neu immunoPET imaging. J Nucl Med. 2009;50:974-981.

43. Mortimer JE, Bading JR, Colcher DM, et al. Functional imaging of human epidermal growth factor receptor 2-positive metastatic breast cancer using ${ }^{64} \mathrm{Cu}$-DOTAtrastuzumab PET. J Nucl Med. 2014;55:23-29. 
44. Dijkers EC, Oude Munnink TH, Kosterink JG, et al. Biodistribution of ${ }^{89} \mathrm{Zr}$-trastuzumab and PET imaging of HER2-positive lesions in patients with metastatic breast cancer. Clin Pharmacol Ther. 2010;87:586-592.

45. Gebhart G, Lamberts L, Garcia C, et al. PET/CT with ${ }^{89} \mathrm{Zr}$-trastuzumab and ${ }^{18} \mathrm{~F}$-FDG to individualize treatment with trastuzumab emtansine (T-DM1) in metastatic HER2positive breast cancer (mBC) [abstract]. J Clin Oncol 2014;32:5(suppl):11001.

46. Gralow JR, Burstein HJ, Wood W, et al. Preoperative therapy in invasive breast cancer: pathologic assessment and systemic therapy issues in operable disease. J Clin Oncol. 2008;26:814-819.

47. Liedtke C, Mazouni C, Hess KR, et al. Response to neoadjuvant therapy and long-term survival in patients with triple-negative breast cancer. J Clin Oncol. 2008;26:1275-1281.

48. von Minckwitz G, Untch M, Blohmer JU, et al. Definition and impact of pathologic complete response on prognosis after neoadjuvant chemotherapy in various intrinsic breast cancer subtypes. J Clin Oncol. 2012;30:1796-1804.

49. Humbert $\mathrm{O}$, Cochet $\mathrm{A}$, Coudert B, et al. Role of positron emission tomography for the monitoring of response to therapy in breast cancer. Oncologist. 2015;20:94-104.

50. Cortazar P, Zhang L, Untch M, et al. Pathological complete response and longterm clinical benefit in breast cancer: the CTNeoBC pooled analysis. Lancet. 2014;384:164-172.

51. Avril S, Muzic RF, Plecha D, Traughber B, Vinayak S, Avril N. ${ }^{18}$ F-FDG PET/ CT for monitoring of treatment response in breast cancer. J Nucl Med. 2016;57 (suppl 1):34S-39S

52. Groheux D, Majdoub M, Sanna A, et al. Early metabolic response to neoadjuvant treatment: FDG PET/CT criteria according to breast cancer subtype. Radiology. April 27, 2015 [Epub ahead of print].

53. Zervoudis S, Peitsidis P, Iatrakis G, et al. Increased levels of tumor markers in the follow-up of 400 patients with breast cancer without recurrence or metastasis: interpretation of false-positive results. J BUON. 2007;12:487-492.

54. Reinventing the Regulation of Cancer Drugs: Accelerating Approval and Expanding Access. Washington, DC: National Performance Review; 1996.

55. Miller AB, Hoogstraten B, Staquet M, Winkler A. Reporting results of cancer treatment. Cancer. 1981;47:207-214.

56. Therasse P, Arbuck SG, Eisenhauer EA, et al. New guidelines to evaluate the response to treatment in solid tumors: European Organization for Research and Treatment of Cancer, National Cancer Institute of the United States, National Cancer Institute of Canada. J Natl Cancer Inst. 2000;92:205-216.

57. Eisenhauer EA, Therasse P, Bogaerts J, et al. New response evaluation criteria in solid tumours: revised RECIST guideline (version 1.1). Eur J Cancer. 2009;45:228-247.

58. Barrington SF, Mikhaeel NG, Kostakoglu L, et al. Role of imaging in the staging and response assessment of lymphoma: consensus of the International Conference on Malignant Lymphomas Imaging Working Group. J Clin Oncol. 2014;32:3048-3058.

59. Erasmus JJ, Gladish GW, Broemeling L, et al. Interobserver and intraobserver variability in measurement of non-small-cell carcinoma lung lesions: implications for assessment of tumor response. J Clin Oncol. 2003;21:2574-2582.

60. Van den Abbeele AD, Badawi RD. Use of positron emission tomography in oncology and its potential role to assess response to imatinib mesylate therapy in gastrointestinal stromal tumors (GISTs). Eur J Cancer. 2002;38(suppl 5):S60-S65.

61. Shepherd FA, Rodrigues Pereira J, Ciuleanu T, et al. Erlotinib in previously treated non-small-cell lung cancer. $N$ Engl J Med. 2005;353:123-132.

62. Sachpekidis C, Larribere L, Pan L, Haberkorn U, Dimitrakopoulou-Strauss A, Hassel JC. Predictive value of early ${ }^{18} \mathrm{~F}-\mathrm{FDG}$ PET/CT studies for treatment response evaluation to ipilimumab in metastatic melanoma: preliminary results of an ongoing study. Eur J Nucl Med Mol Imaging. 2015;42:386-396.

63. A predictive model for aggressive non-Hodgkin's lymphoma: The International Non-Hodgkin's Lymphoma Prognostic Factors Project. N Engl J Med. 1993;329:987-994.

64. Buyse M, Thirion P, Carlson RW, Burzykowski T, Molenberghs G, Piedbois P. Relation between tumour response to first-line chemotherapy and survival in advanced colorectal cancer: a meta-analysis-Meta-Analysis Group in Cancer. Lancet. 2000;356:373-378.

65. Burzykowski T, Buyse M, Piccart-Gebhart MJ, et al. Evaluation of tumor response, disease control, progression-free survival, and time to progression as potential surrogate end points in metastatic breast cancer. J Clin Oncol. 2008;26:1987-1992.
66. Kurihara H, Shimizu C, Miyakita Y, et al. Molecular imaging using PET for breast cancer. Breast Cancer. April 28, 2015 [Epub ahead of print].

67. Weber WA. Assessing tumor response to therapy. J Nucl Med. 2009;50(suppl 1): $1 \mathrm{~S}-10 \mathrm{~S}$.

68. Jacene HA, Leboulleux S, Baba S, et al. Assessment of interobserver reproducibility in quantitative ${ }^{18} \mathrm{~F}$-FDG PET and CT measurements of tumor response to therapy. J Nucl Med. 2009;50:1760-1769.

69. Delbeke D, Schoder H, Martin WH, Wahl RL. Hybrid imaging (SPECT/CT and PET/CT): improving therapeutic decisions. Semin Nucl Med. 2009;39: $308-340$.

70. Cook GJ, Azad GK, Goh V. Imaging bone metastases in breast cancer: staging and response assessment. J Nucl Med. 2016;57(suppl 1):27S-33S.

71. Du Y, Cullum I, Illidge TM, Ell PJ. Fusion of metabolic function and morphology: sequential ${ }^{18} \mathrm{~F}$-fluorodeoxyglucose positron-emission tomography/computed tomography studies yield new insights into the natural history of bone metastases in breast cancer. J Clin Oncol. 2007;25:3440-3447.

72. Tateishi U, Gamez C, Dawood S, Yeung HW, Cristofanilli M, Macapinlac HA. Bone metastases in patients with metastatic breast cancer: morphologic and metabolic monitoring of response to systemic therapy with integrated PET/CT. Radiology. 2008;247:189-196.

73. Avril N, Rose CA, Schelling M, et al. Breast imaging with positron emission tomography and fluorine-18 fluorodeoxyglucose: use and limitations. J Clin Oncol. 2000;18:3495-3502.

74. Dashevsky BZ, Goldman DA, Parsons M, et al. Appearance of untreated bone metastases from breast cancer on FDG PET/CT: importance of histologic subtype. Eur J Nucl Med Mol Imaging. 2015;42:1666-1673.

75. Huyge V, Garcia C, Alexiou J, et al. Heterogeneity of metabolic response to systemic therapy in metastatic breast cancer patients. Clin Oncol ( $R$ Coll Radiol). 2010;22:818-827.

76. Morris PG, Lynch C, Feeney JN, et al. Integrated positron emission tomography/ computed tomography may render bone scintigraphy unnecessary to investigate suspected metastatic breast cancer. J Clin Oncol. 2010;28:3154-3159.

77. Cachin F, Prince HM, Hogg A, Ware RE, Hicks RJ. Powerful prognostic stratification by ${ }^{18} \mathrm{~F}$-fluorodeoxyglucose positron emission tomography in patients with metastatic breast cancer treated with high-dose chemotherapy. J Clin Oncol. 2006;24:3026-3031.

78. Reidl C, Pinker K, Ong L, et al. FDG-PET/CT versus contrast-enhanced CT for prediction of progression-free and disease-specific survival in stage IV breast cancer patients [abstract]. J Clin Oncol. 2015;33(suppl):1051.

79. Mayer IA, Abramson VG, Isakoff SJ, et al. Stand up to cancer phase Ib study of pan-phosphoinositide-3-kinase inhibitor buparlisib with letrozole in estrogen receptorpositive/human epidermal growth factor receptor 2-negative metastatic breast cancer. J Clin Oncol. 2014;32:1202-1209.

80. Lin N, Guo H, Yap J, et al. Phase II study of lapatinib in combination with trastuzumab in patients with human epidermal growth factor receptor 2-positive, metastatic breast cancer: clinical outcomes and predictive value of early ${ }^{18} \mathrm{~F}$-fluorodeoxyglucose positron emission tomography imaging (TBCRC 003). J Clin Oncol. July 13, 2015 [Epub ahead of print].

81. McShane LM, Altman DG, Sauerbrei W, Taube SE, Gion M, Clark GM. Reporting recommendations for tumor marker prognostic studies. J Clin Oncol. 2005;23: 9067-9072.

82. Wahl RL, Jacene H, Kasamon Y, Lodge MA. From RECIST to PERCIST: evolving considerations for PET response criteria in solid tumors. J Nucl Med. 2009;50(suppl 1):122S-150S.

83. van Kruchten M, de Vries EG, Glaudemans AW, et al. Measuring residual estrogen receptor availability during fulvestrant therapy in patients with metastatic breast cancer. Cancer Discov. 2015;5:72-81.

84. Wang Y, Ulaner GA, Manning HC, et al. Validation of target engagement using ${ }^{18} \mathrm{~F}$-fluoroestradiol PET in patients undergoing therapy with selective estrogen receptor degrader, ARN-810/GDC-0810 [abstract]. J Nucl Med 2015;56 (suppl):565.

85. McShane LM, Hayes DF. Publication of tumor marker research results: the necessity for complete and transparent reporting. J Clin Oncol. 2012;30: 4223-4232. 